\title{
A modified formula for defining tissue phantom ratio of photon beams
}

\author{
M. Jahangir Alam ${ }^{1}$, K.S. Rabbani ${ }^{1}$, G.A. Zakaria ${ }^{2}$, S.M. Akram Hussain ${ }^{3}$, \\ Adnan Kiber ${ }^{4}$ and Tanvir Baig ${ }^{1}$ \\ ${ }^{1}$ Department of Physics, University of Dhaka, Dhaka; ${ }^{2}$ Gummerbeach, Cologne University Teaching Hospital, \\ Gummerbeach, Germany; ${ }^{3}$ Department of Oncology, Bangabandhu Sheikh Mujib Medical University, Dhaka; \\ ${ }^{4}$ Department of Applied Physics and Electronics, University of Dhaka, Dhaka, Bangladesh.
}

\begin{abstract}
Tissue phantom ratio (TPR), for square fields of various dimensions has been determined at varying depths in water. The dose in water has been measured at a fixed source-to-surface distance (SSD) of $100 \mathrm{~cm}$ and reference depth of $5 \mathrm{~cm}$ for $6 \mathrm{MV}$ photon beam of Siemens Linear Accelerator Primus II in German Cancer Research Center (DKFZ), Heidelberg, Germany. A modified formula has been developed to calculate the TPR value for isocentric treatment. The present article describes the conversion of the measured data values into a comprehensive and consistent data set by the modified formula, that gives the TPR from Percentage Depth Dose (PDD) with depth as a function of field sizes from $10 \mathrm{~mm} \times 10 \mathrm{~mm}$ upto $300 \mathrm{~mm} \times 300 \mathrm{~mm}$ ) and depth (from $0 \mathrm{~mm}$ to $300 \mathrm{~mm}$ ).
\end{abstract}

\section{Introduction}

The Tissue Air Ratio (TAR) concept works well in iso-centric setup for photon energies of cobalt- 60 and below. For mega-voltage $\mathrm{x}$-rays, produced by high energy medical linear accelerator - the TAR concept breaks down, because of difficulties in measuring the "dose to small mass of water in air" at those energies (the size of the required buildup cap for the ionization chamber becomes excessively large). To bypass this problem, the concept of tissue phantom ratio (TPR) was introduced for use in mega-voltage iso-centric setup. For the measurement of depth dose in SSD and SAD method in water and in air ---the PTW water phantom with MP3 interface has been used to drive the Farmer ion chamber of volume $0.01 \mathrm{cc}$. Mephysto software was also used to drive the ion chamber for data acquisition of increment of $1 \mathrm{~mm}$ both laterally and in depth. Sigma Plot 8.0 was adopted to analyze the measured data, and calculate the standard deviation ${ }^{1,2}$.
Normally TPR value was calculated from the measured PDD data without any modification $\mathrm{TPR}_{20,10}$ can also be obtained from the simple relation:

$\mathrm{TPR}_{20,10}=1.2661 \mathrm{PDD}_{20,10-} 0.0595$

Here $\mathrm{PDD}_{20,10}$ is the ratio of the percent depthdoses at $20 \mathrm{~cm}$ and $10 \mathrm{~cm}$ for a field size of $10 \mathrm{~cm}$ $x 10 \mathrm{~cm}$ defined at the phantom surface with an $\mathrm{SSD}$ of $100 \mathrm{~cm}$. The tissue-phantom ratio is formed by the ratio of the absorbed dose $\mathrm{D}_{2}$ at depth $\mathrm{d}$ to the dose $D_{1}$ at reference depth $d_{r}$ (Fig. 1).

$\mathrm{TPR}(\mathrm{d}, \mathrm{B})=\mathrm{D}_{2} / \mathrm{D}_{1}$

It is important to note that in this formulation, the field size refers to the field size at depth $d$. Accordingly, $\mathrm{D}_{3} / \mathrm{D}_{4}=$ TPR $(\mathrm{d}, \mathrm{C})$. The tissuephantom ratio depends on fewer parameters than does the percentage depth dose. In particular, in this definition TPR is independent of the distance from the source. 

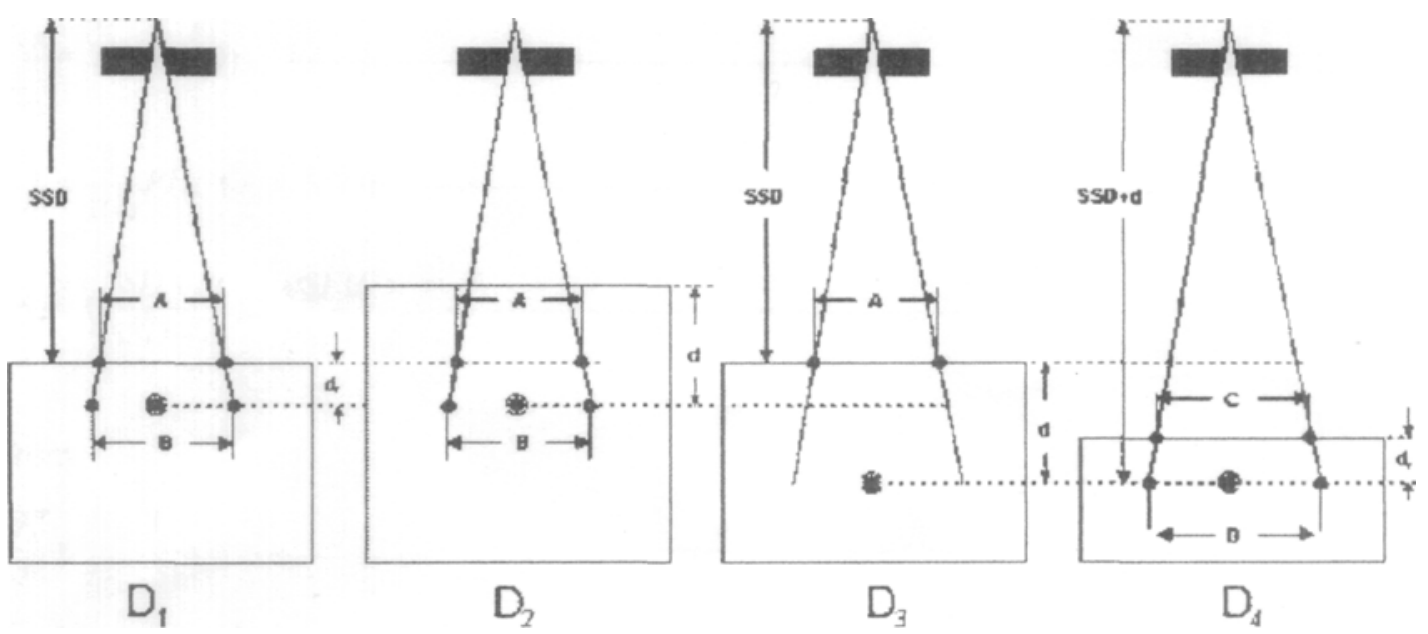

Figure 1: Schematic diagram of the measurement setup to obtain PDD and TPR

In identical system the TPR measurement is carried out using the following formula TPR $\left(\mathrm{z}, \mathrm{A}_{\mathrm{Q}}, \mathrm{h}_{\gamma}\right)=$ Dose rate at $\mathrm{P}$ point $\left(\mathrm{D}_{\mathrm{p}}\right) /$ Dose rate at $\mathrm{P}_{\text {ref }}=$ $\mathrm{Dp} / \mathrm{D}_{\text {pref }}$.

Here $D_{p}$ is measured at an arbitrary depth, where we want to know the TPR value and $D_{\text {ref }}$ is the measured dose at a reference depth of typically 5 $\mathrm{cm}$ on the central beam axis. It is important to mention here that the field size is always constant at point $\mathrm{Q}$ in both the diagrams. The diagram shows that we need to measure the dose at the two different conditions. We need to setup the dosimetry procedure twice for the measurement, thus it has a chance to make an error ${ }^{[2,3]}$. One is due to the setup and another is while filling up or removing water from the water phantom. This is also time consuming. In the work, a modified formula has been developed to measure the TPR value of any field size at any depth from PDD values ${ }^{[4,5]}$. The main advantage of this formulation is that, we can easily measure PDD of any field size at any depth. It has less chance to make mistake, because the ion chamber placement is done once and that is at the central axis of the beam.

\section{The Formulation}

The diagram [Fig.1] of the measurement schemes for PDD and TPR in a water phantom has illustrated their relationship.

$$
\frac{D_{2}}{D_{1}}=\operatorname{TPR}(d, B) .
$$


$\frac{D_{3}}{D_{4}}=T P R(d, D)$

$\frac{D 3}{D 1}=\frac{1}{100} P D D(d, A, S S D)$

$\frac{D_{4}}{D_{1}}=\left(\frac{S S D+d r}{S S D+d}\right)^{2} \cdot \frac{P S F(C)}{P S F(A)}$

From

$\frac{D_{4}}{D_{1}}=\frac{D_{4}}{D_{3}}=\frac{D_{3}}{D_{1}}$

It follows

$\left(\frac{S S D+d r}{S S D+d}\right)^{2} \cdot\left(\frac{P S F(C)}{P S F(A)}\right)=\frac{\frac{1}{100} \operatorname{PDD}(d, A, S S D)}{\operatorname{TPR}(d, D)}$

Using

$B=A \cdot \frac{S S D+d r}{S S D} C=A \cdot \frac{S S D+d-d r}{S S D} D=A \cdot \frac{S S D+d}{S S D}$

One finally obtains

$P D D(d, A, S S D)=100 \cdot T P R\left(d \cdot A \frac{S S D+d}{S S D}\right) \cdot\left(\frac{S S D+d r}{S S D+d}\right)^{2} \cdot \frac{P S F\left(A \frac{S S D+d-d r}{S S D}\right)}{P S F(A)} \ldots$

And

$$
\begin{gathered}
T P R\left(d, A \frac{S S D+d}{S S D}\right)=\frac{1}{100} P D D(d, A, S S D) \cdot\left(\frac{S S D+d}{S S D+d r}\right)^{2} \cdot \frac{P S F(A)}{P S F\left(A \frac{S S D+d-d r}{S S D}\right)} \ldots \ldots \ldots \ldots \ldots \ldots . . .11(a) \text { Or } \\
T P R(d, A)=\frac{1}{100} \cdot P D D\left(d, A \frac{S S D}{S S D+d}, S S D\right) \cdot\left(\frac{S S D+d}{S S D+d r}\right)^{2} \cdot \frac{P S F\left(A \frac{S S D}{S S D+d}\right)}{P S F\left(A \frac{S S D+d-d r}{S S D+d}\right)} \ldots \ldots \ldots \ldots . . .11(b)
\end{gathered}
$$

A complete set of formulas is given to convert the PDD for photon beams from one SSD to another

and to calculate the corresponding central axis quantities, and vice versa.

$$
\operatorname{TPR}(d, A)=\frac{1}{100} \cdot \operatorname{PDD}\left(d, A \frac{S S D}{S S D+d}, \operatorname{SSD}\left(\frac{S S D+d}{S S D+d r}\right)^{2} \cdot \frac{\operatorname{PSF}\left(A \frac{S S D}{S S D+d}\right)}{P S F\left(A \frac{S S D}{S S D+d r}\right)}\right.
$$

The reason for the difference may be due to the assumption used to derive equation 11, that the PSF is independent of $\operatorname{SSD}^{6,7,8}$. This is, however, in reality not true. For consistency reasons, the reader may be referred to the supplement 25 to the British Journal of Radiology if he wants to calculate TPR from PDD. Since the ratio of the peak scatter factors is often very close to unity, this ratio is frequently omitted. Then it does not matter whether equation 11 or 12 is used. 


\section{Results and Discussion}

Figure 2 shows the calculated TPR (CTPR) values obtained by the modified formula versus depth in $\mathrm{mm}$. Directly measured TPR values Vs depth in $\mathrm{mm}$ have been given in Figure 3. Information about the standard deviation of the measured and calculated TPR values are then given in Figure 4.

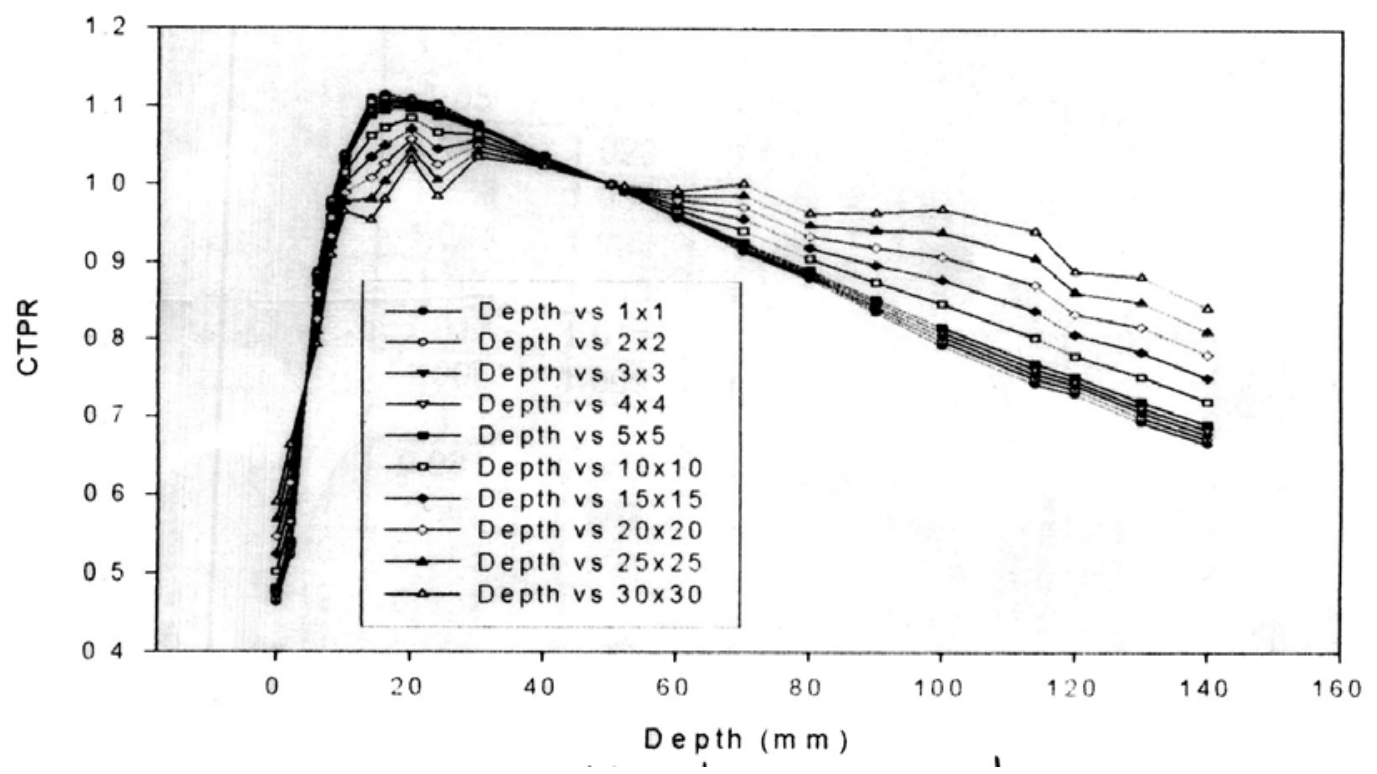

Figure 2: CTPR value obtained by modified formula Vs depth in $\mathrm{mm}$

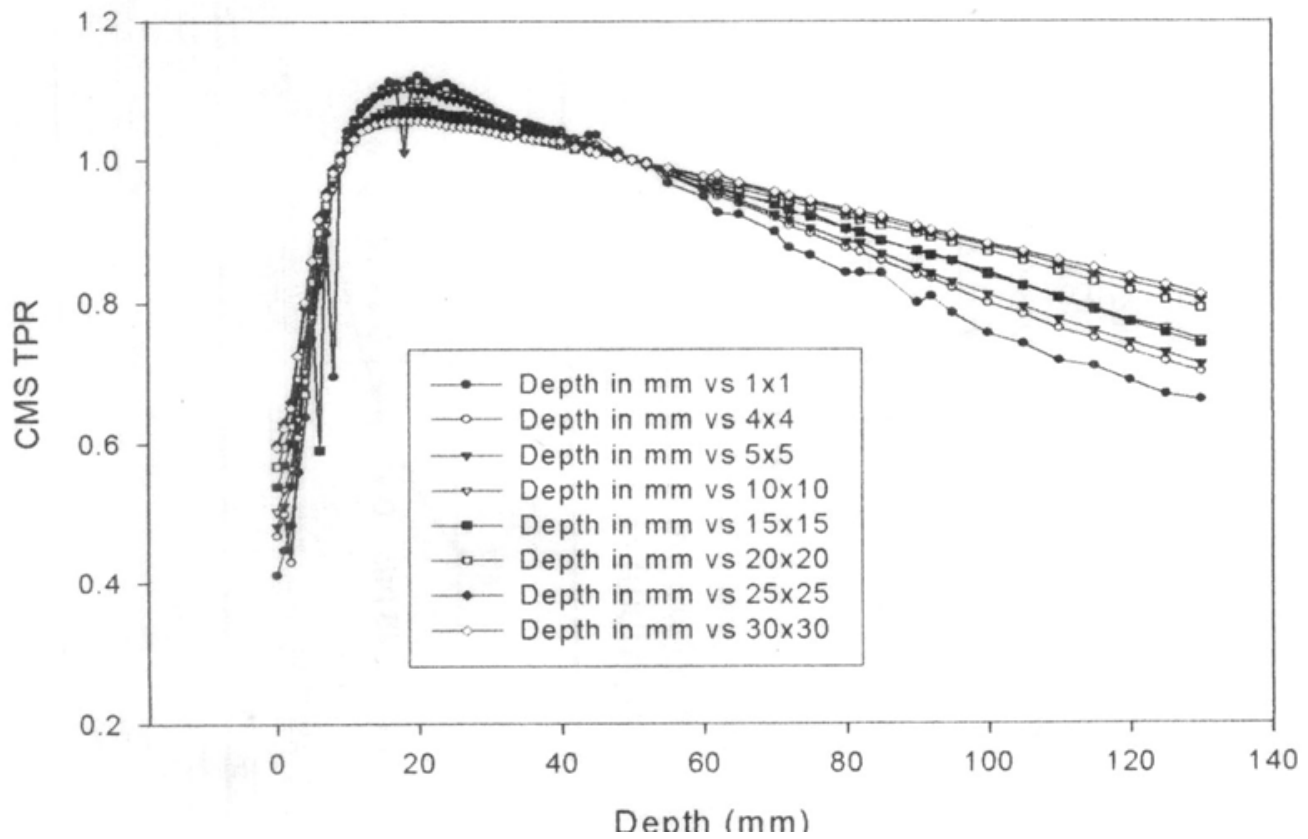

Figure 3: Directly measured TPR value Vs depth in mm 


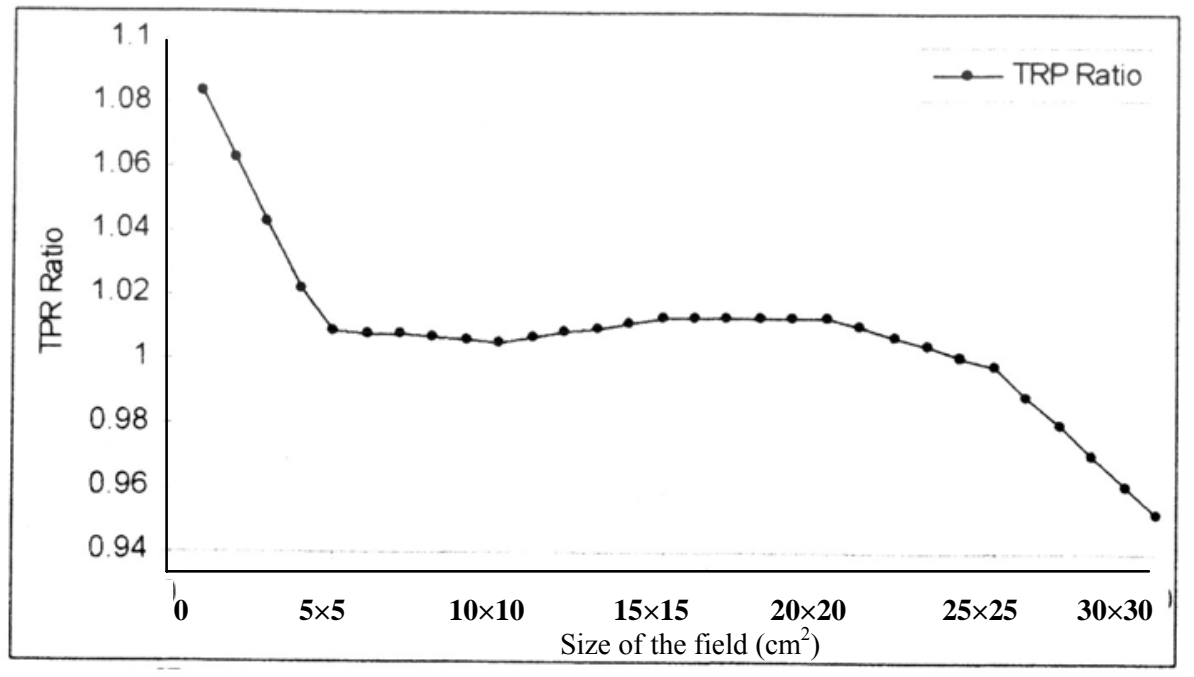

Figure 4: Ratio of standard deviation Vs corresponding field sizes

Several dosimetric functions are available to assist in the computation of absorbed dose in a patient based on the data measured in a phantom. The dosimetric functions describe the doses at various points in space "dressed" with a reference phantom, a phantom or a mini-phantom in air. Iso-centric measurement of ionizing radiation TAR is used only in the energy range of cobalt -60 gamma radiation and below, because of the difficulties of radiation measurement in air. So TPR is the value used in cobalt 60 gamma radiation and in any mega voltage photon beam radiation. It is possible to measure the TPR value in fixed SSD and in SAD formalism. In SAD formalism of measurement of TPR value, it is very difficult to measure it in various depths and for different field sizes. For direct measurement of TPR value of a linear accelerator we need to place the ionization chamber in air and in water at the different environments but in the same setup condition. Two points are worth mentioning here. One is regarding measurement of collimator scattering with mini phantom in air, which has a chance to make an error during setting up the dosimetry system for each and every field size $^{9,10}$. But it is possible to calculate the TPR value in direct measurement of fixed SSD formalism. In fixed SSD formalism PDD measurement is the easiest way and has less chance to make an error, because of the less difficulties in dosimetry setup. If we are able to measure the PDD value in reference condition then it is possible to calculate the TPR value of any field size in any depth.

For TPR data measurement we need to setup the ionization chamber in different conditions in air and in water. The setup will be varied for different field sizes with depth. So the whole procedure is time consuming and has the possibility to loss the consistency of measurement. But it is easy to calculate the TPR value of any field size of different depths in water. If we like to measure the PDD value of the reference condition or of any field size in depth, the formula can be adapted. Another point is that if we normalize the PDD data then it is not mandatory to correct the temperature and pressure.

In conclusion, the modified formulas are applicable to treat cancer, especially for treatment planning in isocentric procedure. This formula has been modified theoretically and is also verified experimentally. That's why one needs to measure the data and put it by export file into the computerized treatment planning system for making a comparison of it by the newly developed formula. Some variation in the data is observed, but it is within the limit. This deviation of the measured and calculated data demands more research work to reduce the difference.

\section{Acknowledgement}

The authors would like to acknowledge German Cancer Research Centre (DKFZ), Heidelberg, Germany and Professor G.H. Hartman, Professor of Medical Physics, DKFZ, Germany for giving one of the authors (MJA), the opportunity to work there. Thanks are also due to Delta Hospital Limited and all technical staffs for rendering the help to analyze the data.

\section{References}

1. Review of radiation oncology physics: A handbook for teachers and students educational reports series. Podgorsak EB (ed). Vienna, International Atomic Energy Agency, 2003. 
2. British Institute of Radiology. Central axis depth dose data for use in radiotherapy. Brit J Radiol. 1996; Suppl. 25 .

3. IAEA. Absorbed dose determination in external beam radiotherapy: An international code of practice for dosimetry based on standards of absorbed dose to water. IAEA, 2000.

4. American Association of Physicists in Medicine (AAPM). AAPM's TG-51 Protocol for clinical reference dosimetry of high energy photon and electron Beams. AAPM Task Group 51 Report. Med Phys. 1999: 26: 1847-70.

5. Pychlau P, Schule E. The exposure rate of a radioactive check device. Phys Med Biol. 1986; 31:1291-93.

6. Christ G. White polystyrene as a substitute for water in high energy photon dosimetry. Med-Phys. 1995; 22: 2097-100.
7. Rhein B, Haring P, Go'lz S, Grosser KH, Moschel IMRT. Verifikation am DKFZ Heidelberg Ein Erfahrungsbericht nach uber 100 IMRT Patienten.ln: Medizinische Physik. Kneschaurek P (ed). 2000, pp 47-48.

8. ICRU. Use of computers in external beam radiotherapy : Procedures with high-energy photons and electrons. ICRU Report 42. Bethesda, International Commission on Radiation Units and Measurements, 1988.

9. ICRU. Tissue substitutes in radiation dosirnetry and measurement. ICRU Report 44. Bethesda, International Commission on Radiation Units and Measurements, 1989.

10. Technical Reports Series No. TRS 430: Commissioning and Quality Assurance of Computerized Planning Systems for Radiation Treatment of Cancer by the IAEA in Austria, 2004. 